\title{
Ueber Haematometra in der verschlossenen unvollkommen entwickelten Hälfte eines Uterus bilocularis.
}

Von

Richard Werth, Kiel.

(Mit 2 Abbildungen im Text.)

In der bekannten Arbeit über Haematometra in dem rudimentären Horn des Uterus unicornis ${ }^{1}$ ) äussert Hegar Zweifel an der Seltenheit dieses Befundes, der ihm in kurzer Zeit zweimal begegnete. Er meint, derartige Haematometren kämen gewiss häufiger vor, nur würden sie nicht richtig erkannt und mit anderen Tumoren verwechselt. Diese Auffassung hat in der Erfahrung der seit jener Veröffentlichung vergangenen zwci Jahrzehnte keine Bcstätigung gefunden. Trotzdem die Casuistik der Uterusmissbildungen seitdem erheblich angeschwollen ist und die Laparotomie in. einer zu jener Zeit ungeahnten Ausdehnung auch zu explorativen Zwecken Verwendung findet, sind - soweit meine Literaturkunde reicht - nur noch zweimal Befunde verzeichnet, die mit den Hegar'schen übereinstimmten. Es ist dies je ein Fall von Leopold (Dieses Arehiv, Bd. 34, S. 377) und von Frankenhäusor (mitgetheilt in der Dissertation von H. Me yer, Zürich, 1883, S. 48). Umgekehrt hat die Zahl. der damals bekannten Fälle von Nebonhornschwangerschaft seitdem einen nicht unbedeutenden Zuwachs erfahren. Haematometra und Schwangerschaft in einem Uterushalbrudiment sind aber Zustände, die nebeneinander nicht bestehen können. Diesen naheliegenden Schluss habe ich zuerst in einer früheren Publication ${ }^{2}$ ) ausgesprochen. Später hat Sänger ${ }^{3}$ ) die

1) Beiträge zur Geburtsh. u. Gynäkol. Bd. III. S. 141. Berlin 1874.

2) Dieses Archiv. Bd. 17. S. 293.

3) Centralbl. f. Gyn. 1888. S. 324. 
gleiche Ansicht geäussert und gegen von Leopold erhobene Einwendungen vertreten. Man darf also wohl auch aus der verhältnissmässigen Häufigkeit der Schwangerschaft im Nebenhorn den. Schluss ziehen, dass das ausgehöhlte Uterushalbrudiment der Regel. nach an der Menstruation nicht Theil nimmt. In dem ersten Hegar'schen Falle hatten die Erscheinungen der Menstruation im abgeschlossenen Nebenhom erst 11 Jahre nach Eintritt der fliessenden Menses begonnen, wahrscheinlich im Anschluss an körperliche Ueberanstrengung. Wegen dieses Umstandes folgerte Hegar, dass die blutige Absonderung in die Höhle des Rudimentes im Anfange fehlen oder wenigstens äusserst gering sein könne, bis durch eine üussere Veranlassung ein starker Congestivzustand zu den Beckenorganen und damit die Blutung hervorgerufen werde ${ }^{1}$ ). In gleichem. Sinne äussern sich P. Müller ${ }^{2}$ ) und Schröder-Hofmeier ${ }^{3}$ ).

Keine Erklärung für dieses Schlummern der menstrualen Function in dem mit einer Höhle versehenen Rudimente, sondern nur eine Analogie zu diesem Verhalten bietet die Erfahrung, dass zuweilen erworbene Atresie im unteren Theile des Uterus eine Unterdrücknng der Menstruation zur Folge hat. Auch bei angeborener Cervixatresie kann Amenorrhoe des vollkommen entwickelten Corpus uteri bestehen. Ich selbst verfüge über eine Beobachtung, welche diese Thatsache demonstrirt und die ich in einem anderen Zusammenhange ausführlicher mitzutheilen beabsichtige.

Bei einer 20jährigen Frau, welche seit 3-4 Jahren typische Molimina hatte, fand sich über einem soliden Cervixrudiment ein wohlansgebildeter Uteruskörper. Die Uterushöhle war bei einer Länge von $3 \mathrm{~cm}$ mit einer sehr hypertrophischen Schleimhaut ausgekleidet, übrigens aber frei von flüssigem Inhalte und von jeder Spur vorausgegangener Blutungen ${ }^{4}$ ).

Es war also nach Allem nicht die diagnostische Unzulänglichkeit Vor- und Gleichlebender, sondern das Gesetz der Duplicität seltenor Fälle, wodurch Hegar dazu gelangte, eine neue Entdeckung auf dem Gebiete der Pathologie uteriner Entwicklungsstörung zu machen.

In der erwähnten Arbeit gedenkt Hegar weiter der Möglich-

1) 1. c. S. 155 .

2) Sterilität der Ehe. Entwicklungsfehler des Uterus. Stuttgart 1885. S. 229 .

3) Handb. d. Krankh. f. weibl. Geschlechtsorgane. 11. Aufl. Leipzig 1893. S. 417 .

4) S. auch Landau und Rbeinstein. Dieses Arch. Bd. 42. S. 276. 
keit, dass auch in der rudimentär entwickelten Hälfte eines Uterus septus einmal Haematometra beobachtet werden könne. Er bezieht sich dabei auf zwei Fälle der Fürst'schen Zusammenstellung ${ }^{1}$ ), welche nach Hegar's Meinung die anatomische Grundlage solcher Möglichkeit darboten. Meiner Ansicht nach sind aber die angezogenenen Fälle in dieser Hinsicht nicht verwerthbar. Die Beschreibung des einen Falles 51, in welchem ein unterer Verschluss der einen Höhle bestand, lässt deutlich erkennen, dass es sich um einen Uterus bicornis mit starker Divergenz der beiden Hörner handelte. In dem anderen Falle, No. 95, lag zweifellos ein Uterus bilocularis vor, dessen eine Hälfte vor nicht langer Zeit geboren hatte und deshalb etwas geräumiger war als die andere. Aber auch in dieser befand sich ein. Uteruscavum von annähernd normalen Dimensionen und mit freier Ausmündung in den ungetheilten Halscanal. Es geht dies deutlich hervor sowohl aus der allerdings etwas unklaren Beschreibung, wie anch aus der beigegebenen schematischen Zeichnung.

Der Uterus bilocularis ist weniger häufig als der bicornis und das Produkt einer Hemmung, wolche das Organ auf einer dem Normalen schon näherliegenden Entwicklungsstufe traf. Deshalb wird halbseitig unvollkommene Entwicklung zusammen mit dieser Form der Doppelbildung gewiss selten - und dem oben Gesagten zufolge Haematometra in dem seitlichen Rudiment eines solchen Uterus erst recht ein ganz ungewöhnlicher Befund sein. Weder vor noch nach 1874 ist meines Wissens ein derartiges Exemplar beschrieben worden. Um so mehr freut es mich, in Folgendem eine Beobachtung vorlegen zu können, die eine, vor so langer Zeit von dem Hochmeister unserer Fachwissenschaft ausgesprochene Voraussetzung endlich bestätigt und anch in diagnostischer wie therapeutischer Hinsicht eine kleine Neuigkeit darstellt. 30. 7. 94 .

J.-N. 15739. W. S., Landmannstochter, 21 J., aufgenommen am

Vater und 5 Geschwister leben und sind gesund. Die Mutter ist im Wochenbette gestorben. Pat. war stets kräftig und gesund, sie ist stets regelmässig menstruirt, mit ziemlich peichlichem, 8 Tage dauendem Blutabgange - von Anfang an mit ziehenden Schmerzen quer über der Schossgegend und im Kreuze, die kurz vor Beginn der Blutung einsetzen und dann in geringem Grade noch mehrere Tage anhalten. Erst seit Januar d. J., wie Pat. meint vielleicht in Folge von Heben einer schweren Last, bestehen anfallsweise aufretende Schmerzen in der

1) Monatsschr. f. Geburtsk. Bd. 30 . 
linken Weiche, die an der Vorderfläche des linken Oberschenkels, etwa bis zu dessen Mitte, ausstrahlen. Die Schmerzen waren gleich viel heftiger als die gewöhnten dysmenorrhoischen und haben bis zur Aufnahme in die Klinik ständig zugenommen. Sie ersehienen häufig und besonders heftig nach körperlicher Thätigkeit, zuweilen jedoch auch Nachts. Nur gelegentlich zeigten sie sich auch zur Zeit der Menstruation, meist unabhängig von dieser und so häufig, dass freie Zwischenräume von mehr als höchstens 3 Tagen nicht vorkamen. Abgesehen von diesen Schmerzen, bestand volllkommene Gesundheit. Seit Januar etwas vermehrter Harndrang ohne Dysurie. Stuhl seit langer Zeit träge. Reichliche Entleerungen auf vom Arzte verordnete Abführmittel liessen die Schmerzen umbeeinflusst. Die letzte Menstruation hatte am 15. Juli begonnen.

St. pr. Kräftig gebautes Hädchen von gut mittlerem Ernährungszustande, gesundem Aussehen, frischen Farben, gut Mittelgrösse.

Gesichtsschädel nicht auffällig breit.

Beckenmaasse: sp. 27,7 ,

er. 31,0 ,

c. e. 19,7 .

Die in Narkose vervollständigte. Genitaluntersuchung ergab folgenden Befund: Portio vagin. von mittlerer Grösse, normaler Form, links stehend. Orif. ext. oval, etwas klein. Rechtes Scheidengewölbe verbreitert, linkes schmal. Portio supra-vaginalis von reichlich normaler Breite und Länge. Corpus uteri über dem vorderen Scheidengewölbe, etwas breit. Rechte Hälfte von normalem Gefüge; die linke von einem ovalen harten Körper eingenommen, der vom Fundus bis nahe an den Isthmus sich erstreckt und dessen seitliche Grenzen nahezu mit der mittleren Theilungsebene des Uterus, resp. der Ansatzlinie des linken Lig. lat. zusammenfallen. Das linke Parametrium ist an der Basis etwas straffer, beide Douglas'schen Falten gut ausgeprägt, symmetrisch nach Länge und Verlauf. Beiderseits mittelgrosse Ovarien in gleichem mittlerem Abstande vom Uterus. Tuben nicht verdickt. (Im wachen Zustande erregte die Betastung der linken Uterushälfte und der nächst anschliessenden Partien des Lig. lat. Sehmerzen).

Speculum: Im Muttermund wenig klares Secret. Sehleimhaut unverändert. Scheidensehleimhaut mässig diffus geröthet. Die 3-mmSonde dringt leicht anf $7 \mathrm{~cm}$ ein. Die Spitze geht nach rechts und lässt sich nicht nach links in den Bereich der linken Uterushälfte resp. des darin liegenden Knotens hinüberführen. Die combinirte Untersuchung bei noch liegender Sonde lässt die Verbindung zwischen der linksseitigen Schwellung und dem übrigen Uterus etwas dünner und beweglicher erscheinen. Drängt man aber den Uterus auf das Rectum und untersucht von hier aus, so erkennt man leicht, dass es sich nur um eine harte, in ihrem ganzen Umkreise in den Grenzen des Uterus eingeschlossene Einlagerung handelt.

Die nachstehende schematische Figur 1 giebt den Tastbefund mit der aus dem Operationsergebniss hervorgehenden Ergänzung: Zusammensetzung der Einlagerung aus einem flüssigen Kerne mit einem verdickten Gewebsmantel.

Meine Diagnose neigte sich von vormherein auf die Seite einer durch Duplicität bedingten Retentionsgeschwulst. Zu einer scharfen Abgrenzung der Diagnose gegenüber anderen entfernten Möglichkeiten 
reichten jedoch die vorhandenen Anhaltspunkte nicht aus. Die letzte Entscheidung musste der Laparotomie vorbehalten bleiben, die als einzig gangbarer Weg bei der Absicht operativen Eingreifens in Frage kam. Letzteres aber war schon deshalb geboten, weil die Kranke wie deren Angehörige für die grossen Beschwerden und die mit diesen verbundene

\section{Fig. 1.}

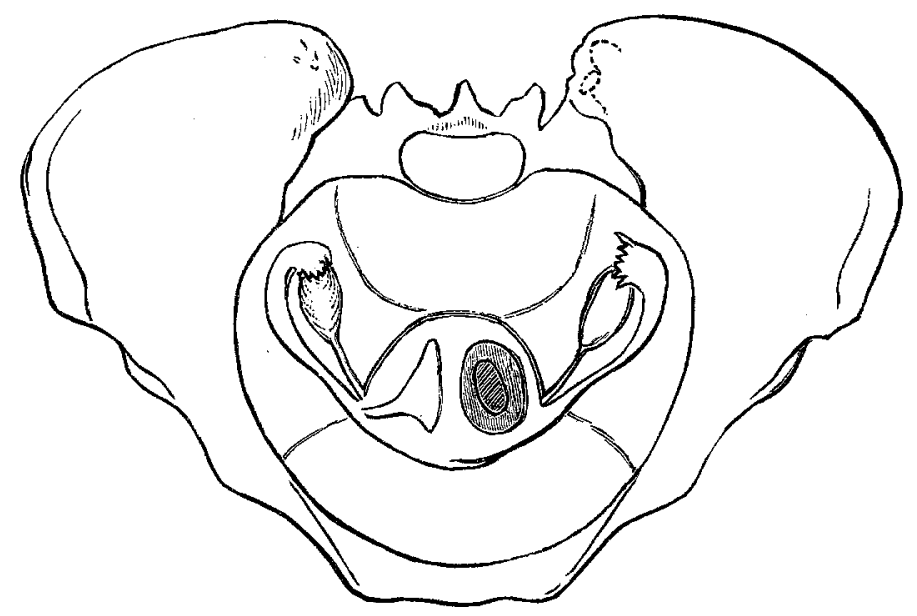

Arbeitsunfähigkeit nach raseher und sicherer Abhülfe verlangten. Die Laparotomie unternahm ich mit der ausgesprochenen Absicht, für den Fall, dass sich meine Diagnose bestätigte, den Uterusabschnitt, welcher die Retentionsgeschwulst enthielt, mit dieser zu reseciren.

4. 8. 94. Laparotomie.

Aethernarkose. Péan-Martin'scher Operationstisch. Ungefähr $10 \mathrm{~cm}$ lange Incision durch den linken Rectus nahe der Linea alba. Der Uterus wird nahe dem linken Tubenursprung, dann in der Mitte des Fundus mit schmaler Muzeux'scher Zange gefasst und mit der Vorderfläche in die Bauchwunde eingestellt. Er erscheint bei beträchtlicher Breite des Corpus von normaler Gestalt. Der Fundus regelmässig ausgewölbt. Die Serosa normal. Der harte Knoten liegt in der Tiefe der linken Uterushälfte, bedingt weder an der vorderen, noch an der hinteren Fläche eine vermehrte Wölbung. Der linke Uterusrand verläuft geradlinig. Die linke Tube trifft den Uterus an normaler Stelle, besitzt einen offenen Pavillon, ist nicht verbreitert, von gleicher Länge wie die rechte, durchaus normal beschaffen. Das linke Lig. lat. nicht verdickt. Beckenserosa überall normal. Ovarien etwas gelappt, von mittlerer Grösse, am normalen Orte. Es wird nun zunächst über der Mitte des Knotens ein kleiner Längsschnitt in der vorderen Uteruswand gemacht. Bis zur Tiefe von ea. $5 \mathrm{~mm}$ durehdringt das Messer völlig gesunde Uterussubstanz, dann kommt ein grauweissliches, etwas derberes Gewebe, bei weiterer Vertiefung des Schnittes ungefähr $1 / 2$ Esslöffel voll alten chokoladenbraunen, etwas schleimigen Blutes.

Es wird nun an der vorderen Fläche links ein mehrere Centimeter breites Oval umschnitten, das oben bis nahe an den Fundus herangeht, 
unten die Grenze der festen Peritonealanheftung nicht ganz erreicht, dem linken Uterusrand um nicht ganz $1 \mathrm{~cm}$ Breite fernbleibt. Die Seitenschnitte dringen steil keilförmig bis auf ca. $1^{1 / 2} \mathrm{~cm}$ Tiefe ein, werden weiterhin flacher geführt und schliesslich mit einander vereinigt. Darauf wird das umschnittene Stück, von unten anfangend, da wo es noch haftet, mit einigen Scheerenschnitten abgetrennt; oben löst es sich bei $\mathrm{Zug}$ von der hier lockeren Uterussubstanz ron selber ab. In der Wunde spritzen nur wenige etwas gröbere arterielle Zweige. Diese werden umstochen. Im Grunde der Wunde ist kein Schleimhantrest zu sehen, nur ziemlich lockeres, normales Muskelgewebe; ebensowenig erscheint die rechtsseitige Uterushöhle eröffnet. Die Wunde wird in doppelter Etage mit dicht liegenden Knopfnähten von Sublimat-Juniperuscatgut vereinigt. Nachdem dies geschehen, erseheint der Uteruskörper von annähernd normaler Breite und regelmässiger Gestalt. Um die etwas schräg vom linken Tubenwinkel nach der Mittellinie hin abwärts verlaufende Nahtlinie gegen die Bauchhöhle abzuschliessen, wird die vordere Uterusfläche durch zwei vom Grunde der Tmschlagsfalte an nach oben bjs zum Fundus sich erstreckende fortlanfende Nähte zn beiden Seiten der Excisionsnaht mit der Blasenserosa vereinigt. Verschluss der Bauchwunde mit besonderer Bauchfell- und Muskelnaht und einigen muskulo-cutanen Seidennähten.

Verlauf: In den ersten Tagen nach der Operation bestanden leichte Blasentenesmen mit halb unwillkürlichem Abgange kleiner Harnmengen - später schmerzlose Entleerung ohne vermehrten Drang. Anfangs vorhandene mässige Schmerzen im Unterleibe hatten bereits am 6. August aufgehört, wo zuerst Flatus abgingen. Vom 7.-11. August bestand eine Blutung, welche ganz wie eine gewöhnliche Menstruation sich verhielt, nur etwas weniger reichlich und gänzlich ohne Schmerzen verlief.

14. 8. Verbandwechsel. Bauchwunde linear verheilt.

20. 8. Untersuchung: Uterus im Ganzen der linken Beckenseite etwas angenähert, rechtwinklig anteflectirt, von annähernd normaler Grösse. An der vorderen Fläche keine Hervorragung zu fühlen. Umgebung normal.

Am 22. 8. verlässt Pat. das Bett, am 25. 8., völlig frei von Beschwerden, die Klinik.

Beschreibung des excidirten Stückes.

Zur genauen Untersuchung des frischen Objectes fehlte es mir an Zeit. Ich musste mich zunächst auf folgende Feststellung beschränken: Das Stück enthält eine kleinkirschgrosse Höhle, die sichtlich gleich nach der Eröffnung sich concentrisch verkleinert hat. Sie ist an der hinteren Schnittfäche des Stückes breit eröffnet. Die Schnittränder sind zackig, die Innenfläche von schleimhantartigem Aussehen, grauroth, mit flachen wulstförmigen Erhöhungen und Vertiefungen. In der Mitte der unteren Schnittfläche verläuft eine $1-2 \mathrm{~mm}$ breite Rinne von dem Schnittrande der Höhle gegen die Serosa hin, ohne diese ganz zu erreichen. Ihr vorderes Ende ist $2-3 \mathrm{~mm}$ von der Serosa entfernt. Kurz vor dem Uebergange in den unteren Schnittrand der Höhle wird die Rinne etwas breiter. Der Grund der Rinne ist in ihrer ganzen Länge bramnroth verfärbt resp. mit einer festhaftenden Schicht alten Blutes bedeckt. Dasselbe ist an der Schleimhaut der Höhle der Fall in nächster Umgebung der Stelle, von wo die Rinne ausgeht. 
Das ausgeschnittene Stiick wurde nach der ersten Besichtigung in Alkohol von steigender Concentration gehärtet und nachdem es bereits eine Reihe von Tagen in absolutem Alkohol gelegen hatte, am 15. Aug. genaner untersucht.

Das Stück erscheint nun, abgesehen von der durch die Härtung bedingten Verkleinerung auch in seiner Form verändert. Die vordere, von Serosa bedeckte Fläche hat sich fast zu einem Quadrat verkürzt, während der ausgeschnittene Conus sichtlich an Tiefe gewonnen hat. Die beigefügte Skizze habe ich von dem gehärteten Stücke entworfen, ebenso sind die unten folgenden Maasse von diesem genommen.

Fig. 2.

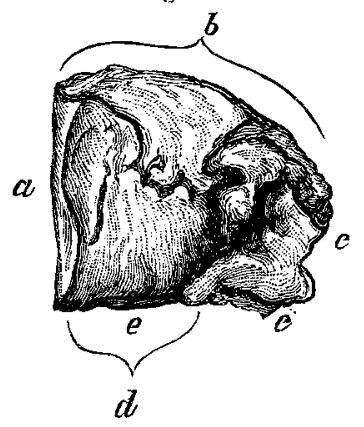

Im Profil erscheint das Stück als abgestutzter, etwas schräg gerichteter Kegel. Die von der vorderen Wandserosa (a) gebildete Grundfläche misst $21 \mathrm{~mm}$ in der Länge, $20 \mathrm{~mm}$ in der Breite. Die dem Fundus entsprechende Schnittfläche (b) verläuft in flachem Bogen in der Länge von $25 \mathrm{~mm}$ zu der bei der Operation eröffneten, im verjüngten Theile des Kegels gelegenen Höhle. Die Schwittwunden der letzteren haben sich in zwei Drittel ihres Umkreises kelchartig nach aussen umgelegt (c). Die untere Schnittfläche des Stïckes (d) stösst in rechtem Winkel auf den unteren Rand des Serosaviereckes. An dieser Fläche befindet sich die oben erwähnte Blutrinne (e).

Der im Stiicke enthaltene Hohlraum hat nach vorn die Gestalt eines Trichters. dessen Spitze $9 \mathrm{~mm}$ von der Serosa entfernt ist.

Ein etwa dem interstitiellen Abschnitte der Tube entsprechender Gang lässt sich an dem gehärteten Objecte nicht auffinden.

Für die mikroskopische Untersuchung wurde das im Ganzen gehärtete Stück dureh einen ungefähr der Mitte der Höhle entsprechenden Horizontalschnitt halbirt, dann wurden parallel der Schnittfläche dureh die ganze Dicke des Stückes gehende Mikrotomsehnitte angefertigt. Deren Betrachtung ergab das Vorhandensein eines einschichtigen Cylinderepithels über ungefähr zwei Drittel des Höhlenumfanges. Der Ḧ̈hlencontur verläuft theils gradlinig, theils zackig, stellenweise in regelmässig kurzwelliger Linie, hier und da schneidet er auch etwas tiefer in schmaler Spaltform in das Stroma ein. Typische Drüsen sind nirgends zu finden, nur an wenigen Stellen erinnern niedrige flasehenförmige Einbuchtungen an die Drüsenanlagen in der Uterusschleimhaut junger Kinder. Das Epithel ist besonders da, wo der freie Schleimhautrand gestreckten Verlauf hat, ziemlich breit und von geringer Höhe, vielfach aber hoch 
und schmal, von Kelehglasform. Ueber den gewölbten Abschnitten des Schleimhautrarides zeigen diese hohen schmalen Epithelzellen vielfach büschelförmige Anordnung.

Die innerste subepitheliale Schicht besteht aus polygonalen ziemlich grossen Zellen, die von den Stromazellen der normalen Uterusschleimhaut sich kaum unterscheiden, in der Tiefe - zuweilen auch schon nahe dem Epithel - sich der Spindelform nähern. Diese Sehicht ist von sehr ungleicher, im Ganzen äusserst geringer, an manchen Orten nur auf wenige Zelllagen beschränkter Breite.

Die subepitheliale Sehicht ist gegen die unterliegende Muscularis sehr unregelmässig und wenig scharf begrenzt, da vielfach Abzweigungen der Muskelschicht in sie eindringen und auf der anderen Seite an Stelle des sonst fibrillären Bindegewebes Fortsätze der zelligen Stromaschicht zwischen die Bündel der angrenzenden Muscularis sich einschieben.

Hier und da findet sich die subepitheliale Zellsehicht von noch etwas grösseren rundlichen Elementen durchsetzt, die ein stark körniges und stark gelb gefärbtes Protoplasma besitzen. Die gleichen Elemente finden sich in kleineren Haufen auch zwischen den Bündeln der oberflächlichen Muskelzone. - An einzelnen Partien der inneren Auskleidungsschicht sind die Stromazellen etwas auseinander gedrängt und die Zwischenräume eingenommen von gelben körnigen Massen - zerfallenen Erythrocyten. Die Anordnung der Muskulatur ist in grosser Ausdehnung um die Höhle herum eine röllig regellose, indem die Huskelbündel in den verschiedensten Richtungen sich kreuzen. In einer ziemlich breiten, der Höhle nächst gelegenen Zone ist die Muskulatur etwas dichter, ärmer an Bindegewebe, auch erscheinen besonders im Querschnitt die Muskelzellen etwas grösser als in der peripheren Schicht.

Aus mikroskopischen Schnitten, welche senkrecht zu der Blutrinne durch die Muskulatur entsprechend der unteren Schnittfläche des Stückes geführt waren, ersieht man Folgendes:

Im Grunde der Rinne liegt eine körnige gelbe Masse, welche einzeln liegende grosse wie gequollene Zellen einschliesst mit verhältnissmässig kleinem, schlecht gefärbtem Kerne. Gleichzeitig ist diese Schicht von Streifen hyalin gequollenen, gefärbte Kerne nicht mehr enthaltenden Bindegewebes (diffuse Rothfärbung bei Tinction nach van Gieson) durchzogen, die aus der unveränderten fibrillären Bindesubstanz der an die Rinne angrenzenden Gewebszone hervorgehen. In dieser Zone erscheint die Muscularis lückenhaft, ihr Balkenwerk von breiteren Zwischenräumen durchsetzt, in welchen zwischen spärlichen Fibrillennetzen dicht gedrängt z. Th. Rundzellen von dem Charakter einkerniger Leukocyten, meist aber grosse endothelioide Zellen liegen. Thr Protoplasma ist zum Theil stark gekörnt und gelb gefärbt. Innerhalb dieser Zellherde sind vielfach erweiterte Capillaren sichtbar.

Noch in ziemlich weiter Entfernung von der Blutrinne fällt ein abnormer Zellgehalt des intermuskulären Bindegewebes, namentlich in der nächsten Ümgebung der Blutgefässe auf.

Zur der vorstehend mitgetheilten Beobachtung mögen noch einige epikritische Bemerkungen hier ihre Stelle finden.

Ein Zweifel an der Richtigkeit der anatomisehen Diagnose scheint mir beim besten oder vielmehr schlechtesten Willen nicht möglich. Der ausgeschnittene Hohlraum lag central in der linken Uterushälfte. 
Er war zu einem grossen Theil mit einem typischen Cylinderepithel - wenn auch von wechselnder Höhe - ausgekleidet. Obwohl das ausgeschnittene Stück nahezu die ganze linke Hälfte des Corpus uteri ausmachte, wurde bei der Ausschneidung desselben die in den Cervicalcanal mündende Uterushöhle nicht eröffnet; sie lag also ausschliesslich in der rechten Hälfte des Organes. Der Mangel voll ausgebildeter Drüsen entspricht nur dem rudimentären Zustande der linksseitigen Körperhöhle und steht in Einklang mit dem Befunde an der Mehrzahl der Uteri im Fötalleben resp. frühesten Kindesalter. Auffallend scheint zunächst, dass die Kanalisation der linken Hälfte über einen rudimentären Ansatz nicht hinaus gelangt war, während doch der äusseren Form und der Massenentwicklung der linken Hälfte nach der Uterus dem Bilde eines vollausgebildeten Uterus bilocularis entsprach. Dieser Widerspruch erklärt sich nur zum kleineren Theile aus dem immerhin nur geringen Zuwachs an Umfang, welcher aus der nicht bedeutenden Ausdehnung des Höhlenrudiments sich ergab. Zweifellos war auch der Gehalt an Muskelmasse auf der linken Seite gegenüber der anderen ursprünglich in erheblichem Rückstande, bis die Ansammlung von Menstrualblut in der verschlossenen Höhle eine Hypertrophie des Muscularis hervorrief und damit die in der Anlage vorhandene Differenz zum Ausgleich brachte.

Eine Folgerung, welche der von mir geschilderte Befund naherückt, möchte ich hier nur flüchtig streifen. Dieser Befund eröffnet die Aussicht auf eine bisher noch nicht beschriebene oder nicht erkannte Form der ectopischen. Schwangerschaft, welche der interstitiellen Tubenschwangerschaft in formaler Beziehung ausserordentlich nahe stehen würde.

Der Ausspruch Hegar's, dass für die Erkenntniss der Hämatometra im Nebenhorn schon die physikalische Untersuchung allein hinreichenden Aufschluss geben müsse, gilt für den hier vorliegenden Fall wohl nieht in gleichem Umfange. Der Blutherd mit der verdichteten muskulären. Grenzschicht erscheint als ein der Uterussubstanz eingelagerter harter Knoten, der für die Untersuchung zunächst kein unterscheidendes Merkmal, z. B. von einem interstitiellen Myome darbietet. Wenn nicht der grosse Unterschied in der Consistenz der beiden Uteruskörperhälften zunächst den Tasteindruck einer doppelhornartigen Gestaltung des Fundus vorgetäuscht hätte, würde wahrscheinlich nicht so unmittelbar sich der Gedanke an eine Bildungsanomalie mir aufgedrängt haben. Dann 
liess mich allerdings die Feststellung excentrischer Lage der Uterushöhle auf der Spur beharren und weitere Ueberlegungen befestigten mich inVerfolgung derselben. Schon dieLage des Herdes liess eine entzündliche (tuberkulöse) Infiltration der Muscularis, die durch Uebergreifen von dem interstitiellen Theil der Tube aus nicht selten zu Stande kommt, ausschliessen, ganz abgesehen davon, dass die Anhänge der linken Seite selber von tastbaren Veränderungen frei waren. Dann blieb nur noch die Möglichkeit, dass es sich um ein Myom handle, offen, eine Annahme, die sowohl mit dem jugendlichen Alter der Kranken wie mit der Heftigkeit der bestehenden Krankheitserscheinungen sich schwer vereinigen. liess.

Der Verwerthbarkeit der Symptome für die Diagnose abträglich und entschieden auffällig war der Umstand, dass die Retentionsschmerzen von der Menstruation zeitlich unabhängig erschienen. Da die Anfälle häufig kamen und die Menge der verhaltenen Flüssigkeit klein war, so darf die Ursache der Schmerzen wohl kaum in etwas Anderem als in periodisch auftretenden Zusammenziehungen des Uterus gesucht werden. Die Schmerzen beschränkten sich im Gegensatze zu den bereits seit der Pubertät bestehenden dysmenorrhoischen. Schmerzen auf das linke Hypogastrium. Deshalb nehme ich an, dass auch nur die hypertrophische Muskulatur der linken Uterushälfte an diesen Contractionen betheiligt war. Der von der linken Uterushälfte eingeschlossene Blutherd unterhielt einen anhaltend gesteigerten Erregungszustand in den dieser Hälfte zugehörenden motorischen Bahnen, der sich in häufig auftretenden Koliken entlud, welche bald spontan, bald dureh den hinzutretenden Reiz körperlicher Anstrengung ausgelöst wurden.

Durch dieses von der Menstruation der offenen Hälfte zeitlich unabhängige Auftreten der Retentionskoliken unterscheidet sich meine Beobachtung von den bisher beobachteten Fällen von Hämatometra in der rudimentären Hälfte eines Uterus bicornis. Hier fielen jedesmal die heftigeren Schmerzanfälle auf den Zeitpunkt der sichtbaren Menstruation. Dagegen ist das späte Auftreten der Retentionserscheinungen meiner Beobachtung mit zweien der oben erwähnten gemeinsam. In dem ersten Hegar'schen Falle waren elf, in dem Leopold'schen gegen sieben Jahre seit Beginn der Menstruation vergangen, bis die Betheiligung des rudimentären Hornes an dieser sich bemerkbar machte. Wie in meinem, so wurde auch in dem Hegar'sehen Falle starke körperliche Anstrengung als Anlass für das erste Auftreten der. Symptome bezeichnet. 
Die Beschwerden, welche in meinem Falle die Menstruation von der Pubertätszeit an begleiteten, waren sicher nur durch die menstrualen Vorgänge in der offenen Uterushälfte bedingt und nichts anderes, als eine gewöhnliche Dysmenorrhoe. An dieser Auffassung kann der Umstand, dass die erste Menstruation nach der Operation völlig schmerzlos verlief, nicht irre machen. Die vorher stattgefundene Sondirung, die Blutentziehung bei der Operation, die anhaltende Bettruhe genügen, diesen Wechsel zu erklären. Hätte auch die linke Seite schon früher menstruirt, so wäre weder die geringe Grösse der linksseitigen Hämatometra noch das so viel spätere Erscheinen auf diese hinweisender Symptome zu verstehen.

Die Prognose der Haematometra steht im Wesentlichen unter dem Zeichen der so leicht sich anschliessenden Tubenblutgeschwulst. Sind nun auch die Trägerinnen einer Haematometra in einer rudimentären Uterushälfte der gleichen Gefahr ausgesetzt?

In den beiden Hegar'schen Beobachtungen, dem Frankenhäuser'schen und meinem Falle erwies sich die zugehörige Tube unbetheiligt, eine bemerkenswerthe Thatsache, wenn wir berücksichtigen, dass die Symptome der Haematometra zum Theil schon längere Zeit, in dem Frankenhäuser'schen Falle bereits seit 11 Jahren, bestanden. Dagegen fand sich in dem Leopold'schen Falle die Tube in der That erkrankt. Ich kann aber gelinde Zweifel an dem ursächlichen Zusammenhange dieser Erkrankung mit der Haematometra nicht unterdrücken. Bei einer ersten, am 17. October 1882 unternommenen Laparotomie wurden die Adnexa auch auf der Seite des mit Blut gefüllten Hornes noch gesund gefunden. Bei der zweiten, nicht ganz sieben Monate später ausgeführten Laparotomie fand sich diese Tube dagegen in einen stark gewundenen, prallen, kleinfingerdicken Wulst verwandelt. Sie hatte eine vornehmlich in der Muscularis stark verdickte Wand mit sammetartig flockiger Schleimhaut, enthielt kein Blut, sondern nur etwas trübröthlichen dicken Schleim. Das Ostium abdominale war nicht verschlossen. Tube und Ovarium fanden sich bei dieser Laparotomie mit dem Horne, dem nächstliegenden Dünndarme und unter sich durch dünne und stärkere Stränge zu einem Knäuel mehrfach verlöthet. An die Explorativlaparotomie hat sich demnach eine ziemlich ausgedehnte locale Peritonitis angeschlossen, und es liegt die Vermuthung nicht allzufern, dass ein Vebergreifen dieser Entzündung auf die Tube die erwähnten Veränderungen 
hervorgerufen hatte. Jedenfalls entspricht die Beschreibung nicht ganz dem Bilde, unter dem wir uns beginnende Haematosalpinx vorstellen, sondern wohl eher dem einer gewöhnlichen Salpingitis. Unter den Frankenhäuser'schen Beobachtungen, welche in der Meyer'schen Dissertation mitgetheilt sind, findet sich noch eine (Fall II), bei der Haematosalpinx neben Haematometra lateralis bestand. Ich habe diesen Fall bisher nicht berücksichtigt, weil es sich nach dem klinisehen und leider zu wenig genau beschriebenen anatomischen Befunde wohl ziemlich sicher nicht um ein Rudiment, sondern ein voll ausgebildetes Uterushorn mit cervicaler Atresie handelte. Dieses hatte nach der durchgängigen Seite hin sich zeitweise eröffnet und war wohl nur wegen dieser öfters erfolgten Entleerungen nicht zu einer Apfelgrösse übersteigenden Ausdehnung gelangt. Uebrigens war noch 15 Tage vor dem Tode ungefähr "ein Schoppen" voll sichtlich aus der Haematometra stammenden Blutes per vaginam abgegangen.

Auch ein von Czerny operirter Fall, den von Meyer mittheilt ${ }^{1}$ ), Haematometra lateralis und Haematosalpinx bei Uterus bicomis und Defect des einen Halses, stand hinsichtlich des primären Entwicklungszustandes des verschlossenen Hornes wohl auf gleicher Stufe wie der eben erwähnte.

Nach Maassgabe der bisher vorliegenden Erfahrungen lässt sich also wohl behaupten, dass die Wahrscheinlichkeit einer secundären Blutanhäufung in der Tube nicht besteht oder sehr gering ist überall, wo die verschlossene Hälfte zur Zeit, da sie zu menstruiren beginnt, den ausgesprochenen Charakter des Rudimentes besitzt, während die Gefahr des Gegentheiles um so näher rïckt, je weniger sich der primäre Entwicklungszustand der verschlossenen Hälfte von dem der wohlausgebildeten offenen Seite unterscheidet.

Die Möglichkeit eines Ausganges, der eine Art Selbsthilfe darstellen kann, lehrt meine Beobachtung kennen. Ich fand einen engen Gang, der von dem unteren Umfange der Bluthöhle bis nahe an die Serosa der vorderen Uteruswand heranführte. Binnen kurzer Zeit wäre eine Peritonealfistel entstanden, welche eine wenigstens theilweise Entleerung des verhaltenen Blutes ermöglicht haben würde. Betrachtungen anzustellen über die möglichen - günstigen oder ungünstigen - Folgen eines so zu Stande gekommenen Durchbruches halte ich für müssig.

1) Deutsche med. Wochenschr. 1893. S. 936. 
434 Werth, Ueber Haematometra in d. versehloss. Hälfte cines Uterus bilocul.

Am Einfachsten stellt sich bei der Haematometra im Seitenrudiment das therapentische Problem. Dass man dem Blutherde direct zu Leibe gehen, wenn irgend möglich nicht bloss den Inhalt, sondern auch das Gefäss beseitigen muss, und dass zu diesem Ziele ausschliesslich der Weg durch die Bauchhöhle führt, dem wird heutzutage schwerlich widersprochen werden ${ }^{1}$ ). Die bisher bekannt gewordenen Beobachtungen liegen schon ziemlich weit zurück, sind zum Theil zu einer Zeit gemacht, wo eingreifende Operationen in die Bauchhöhle mit Recht gefürchtet wurden. In der modernen Aera der Laparotomie würden die Autoren, welchen wir diese Beobachtungen verdanken, schwerlich noch auf ein palliatives, auch als solches unzureichendes und bedenkliches Verfahren, wie es die Punction von der Vagina aus ist, sich beschränkt oder es vorgezogen haben, von jedem Bingriffe abzustehen (Frankenhäuser). Das Gleiche gilt von der Castration, von deren weit über die Geschlechtsphäre hinausgreifenden nachtheiligen Wirkungen uns erst die letzte Zeit bessere Kunde gebracht hat. Auch diese Operation sollte man, nur aus Rücksicht auf die vielleicht etwas geringere Grösse der materiellen Verletzung; der Exstirpation des Blutsackes nicht vorziehen.

Uebrigens dürften technische Schwierigkeiten, soweit die Haematometra im Nebenhorn in Frage kommt, kaum zu erwarten sein. Etwas anders verhält es sich mit Haematometra im Seitenrudiment eines äusserlich einfachen Uterus. Dass auch hier mittelst Resection der den Blutraum abschliessenden Hälfte Abhilfe möglich ist, lehrt die Beobachtung, wclche zu der hier schliessenden Mittheilung Anlass gab.

1) cf. Fritsch, Krankheiten d. Frauen, 5. Aufl., S. 121, and Gläser, Centralbl. f. Gynäkol., 1892, S. 649.

Kiel, im August 1894. 